OPEN ACCESS

Edited by:

Peng Zhang,

Shanghai Institutes for Biological

Sciences (CAS), China

Reviewed by:

Kan Wang,

lowa State University, United States

Eva Stoger

University of Natural Resources and Life Sciences, Vienna, Austria

*Correspondence: Nigel J. Taylor ntaylor@danforthcenter.org

Specialty section: This article was submitted to Plant Biotechnology,

a section of the journal

Frontiers in Plant Science

Received: 21 July 2017 Accepted: 30 September 2017 Published: 18 October 2017

Citation:

Odipio J, Alicai T, Ingelbrecht I, Nusinow DA, Bart $R$ and Taylor NJ (2017) Efficient CRISPR/Cas9 Genome Editing of Phytoene desaturase in Cassava.

Front. Plant Sci. 8:1780. doi: 10.3389/fpls.2017.01780

\section{Efficient CRISPR/Cas9 Genome Editing of Phytoene desaturase in Cassava}

\author{
John Odipio ${ }^{1,2,3}$, Titus Alicai ${ }^{2}$, Ivan Ingelbrecht ${ }^{3,4}$, Dmitri A. Nusinow ${ }^{1}$, Rebecca Bart ${ }^{1}$ and \\ Nigel J. Taylor ${ }^{1 *}$
}

${ }^{1}$ Donald Danforth Plant Science Center, St. Louis, MO, United States, ${ }^{2}$ National Crops Resources Research Institute, Kampala, Uganda, ${ }^{3}$ Vlaams Instituut voor Biotechnologie, Department of Plant Biotechnology and Bioinformatics, Faculty of Sciences, Ghent University, Ghent, Belgium, ${ }^{4}$ FAO/IAEA Division of Nuclear Techniques in Food and Agriculture, Department of Nuclear Sciences and Applications, International Atomic Energy Agency, Vienna, Austria

CRISPR/Cas9 has become a powerful genome-editing tool for introducing genetic changes into crop species. In order to develop capacity for CRISPR/Cas9 technology in the tropical staple cassava (Manihot esculenta), the Phytoene desaturase (MePDS) gene was targeted in two cultivars using constructs carrying gRNAs targeting two sequences within MePDS exon 13. After Agrobacterium-mediated delivery of CRISPR/Cas9 reagents into cassava cells, both constructs induced visible albino phenotypes within cotyledon-stage somatic embryos regenerating on selection medium and the plants regenerated therefrom. A total of 58 (cv. 60444) and 25 (cv. TME 204) plant lines were recovered, of which 38 plant lines (19 from each cultivar) were analyzed for mutagenesis. The frequency of plant lines showing albino phenotype was high, ranging from 90 to $100 \%$ in CV. TME 204. Observed albino phenotypes were comprised of full albinos devoid of green tissue and chimeras containing a mixture of white and green tissues. Sequence analysis revealed that 38/38 (100\%) of the plant lines examined carried mutations at the targeted MePDS site, with insertions, deletions, and substitutions recorded. One putatively mono-allelic homozygous line (1/19) was found from cv. 60444, while 1 (1/19) and 4 (4/19) putatively bi-allelic homozygous lines were found in 60444 and TME204, respectively. The remaining plant lines, comprised mostly of the chimeras, were found to be putatively heterozygous. We observed minor (1 bp) nucleotide substitutions and or deletions upstream of the $5^{\prime}$ and or downstream of the $3^{\prime}$ targeted MePDS region. The data reported demonstrates that CRISPR/Cas9-mediated genome editing of cassava is highly efficient and relatively simple, generating multi-allelic mutations in both cultivars studied. Modification of MePDS described here generates visually detectable mutated events in a relatively short time frame of 6-8 weeks, and does not require sequencing to confirm editing at the target. It therefore provides a valuable platform to facilitate rapid assessment and optimization of CRISPR/Cas9 and other genome-editing technologies in cassava.

Keywords: cassava, genome editing, CRISPR/Cas9, Phytoene desaturase (PDS), albino, mutation, homozygous, heterozygous 


\section{INTRODUCTION}

Development of superior crop varieties has relied on securing genetic gains through sexual recombination, induced random mutagenesis, and transgenic approaches. The recent emergence of targeted genome-editing technologies offers a new avenue for incorporating beneficial genetic changes in the world's most important crop species (Zhang et al., 2017).

Gene-editing technologies based on zinc-finger nucleases (ZFNs), transcription activator-like effector nucleases (TALENs), and clustered regularly interspaced short palindromic repeats (CRISPR) make use of engineered nucleases to induce double strand breaks (DSB) at known DNA sequences within the genome. Subsequent repair at the target site introduces variation via error prone non-homologous end joining (NHEJ) (Sonoda et al., 2006; Curtin et al., 2012; Joung and Sander, 2013) to generate insertions and deletions (INDELs), with occasional substitution of nucleotides occurring at the repair regions. Alternatively, the error free homologous recombination (HR) repair pathway can be activated in presence of a homologous donor DNA template spanning the DSB, resulting in targeted gene replacement (Puchta et al., 1996; Johnson and Jasin, 2001; Symington and Gautier, 2011).

The ZFNs and TALENs are based on protein-guided recognition mechanisms. Vector construction for targeting DNA sequences using these technologies is a relatively complex and costly process. In contrast, the CRISPR/Cas9 system depends on DNA or RNA sequence homology, in which targeting a specific DNA sequence requires only a 17-23-bp complementary nucleotide sequence called a guide RNA (gRNA). This versatility, plus high efficacy, and low cost have made CRISPR/Cas9 the most widely adopted of the three genome-editing technologies (Doudna and Charpentier, 2014). CRISPR/Cas9 has been applied to delete, activate, and suppress targeted genes in human cells, animals, and plants (Pennisi, 2013). Plant species altered by CRISPR/Cas9 technology include Nicotiana benthamiana (Nekrasov et al., 2013), Arabidopsis, tobacco, sorghum, and rice (Jiang et al., 2013), tomatoes (Brooks et al., 2014), wheat (Shan et al., 2013), soybean (Sun et al., 2015), maize (Svitashev et al., 2016), potato (Wang et al., 2015), sweet orange (Jia and Wang, 2014), populus (Fan et al., 2015), cucumber (Chandrasekaran et al., 2016), and cotton (Li et al., 2017). In several cases, CRISPR/Cas9-induced mutations have been confirmed to be heritable across sexual generations (Brooks et al., 2014; Ding et al., 2016).

Cassava (Manihot esculenta Crantz) is an essential source of calories for millions of people across the world's tropical regions, providing storage roots for local consumption and raw materials for processed foods and industrial products (Ceballos et al., 2015). Conventional breeding for cassava improvement is constrained by its long life cycle, high level of heterozygosity, poor flowering, and seed set and inbreeding depression (Ceballos et al., 2004). CRISPR/Cas9-targeted genome editing therefore offers important new opportunities for addressing pre- and postharvest constraints affecting cassava production and utilization. To evaluate the potential of CRISPR/Cas9 in a new species such as cassava, a reproducible system for design, construction, and delivery of Cas9/gRNA reagents must be developed and validated. We report here an efficient CRISPR/Cas9-mediated targeting of Phytoene desaturase (PDS) in two cassava cultivars. The results reported set the groundwork for future studies of gene function and trait development using the CRISPR/Cas9 gene-editing technology in this important staple food crop.

\section{MATERIALS AND METHODS}

\section{In Silico Identification of Cassava Phytoene desaturase and CRISPR/Cas9/gRNA Vector Construction}

BLAST was used to search the reference cassava genome in Phytozome 11 (version 4.1) ${ }^{1}$ for nucleotide sequences homologous to the Arabidopsis thaliana PDS gene (AtPDS: NM117498). A single copy gene, here referred to as MePDS (Manes.05G193700/cassava4.1_004359m.g), was identified. The CRISPR-P program (version 1$)^{2}$ was employed to design gRNAs. Two target sequences were selected by initially identifying the NGG protospacer adjacent motif (PAM) sequence essential for Streptococcus pyogenes Cas9, followed by 20 nucleotides upstream of the PAM sequence for use as the spacer in the gRNA to target MePDS. Two gRNAs chosen were located in the $13^{\text {th }}$ exon, each having a $G$ at their $5^{\prime}$ end to enhance gRNA transcription in plant cells using the U6-26 promoter when transcribed as single molecule with gRNA scaffold (Figures 1A,C).

A pair of complementary DNA oligonucleotides were synthesized (Integrated DNA Technologies ${ }^{3}$ ) and hybridized to form a duplex which has compatible ends with the BsaI-digested empty vector (pCAMBIA2300_CR3-EF, provided by B. Staskawicz, University of California, Berkeley, CA, United States). The empty vector construct contained Cas9 coding sequence from $S$. pyogenes and gRNA scaffold with a BsaI restriction site for insertion of a single gRNA. The included nptII marker gene aids in selection of transgenic cells during regeneration process. The $35 \mathrm{~S}$ Cauliflower mosaic virus promoter was utilized to drive expression of Cas9, while the U6-26 promoter from Arabidopsis drove expression of the gRNAs (Figure 1C). The presence of the inserted gRNA and stability of the final constructs was confirmed by sequencing. The two binary vectors, MePDS-1 and MePDS-2, each carrying one gRNA, were transformed into Agrobacterium strain LBA 4404 by electroporation.

\section{Cassava Transformation and Visual Confirmation of PDS Mutants}

Friable embryogenic callus (FEC) was generated from cassava cultivars 60444 and TME 204 and used as target tissue for transformation with the gene-editing constructs MePDS-1 and MePDS-2, plus empty vector (EV) and green fluorescent protein (GFP) controls (Taylor et al., 2012; Chauhan et al.,

\footnotetext{
${ }^{1}$ https://phytozome.jgi.doe.gov/pz/portal.html

${ }^{2}$ http://cbi.hzau.edu.cn/cgi-bin/CRISPR

${ }^{3}$ https://www.idtdna.com/site
} 
A

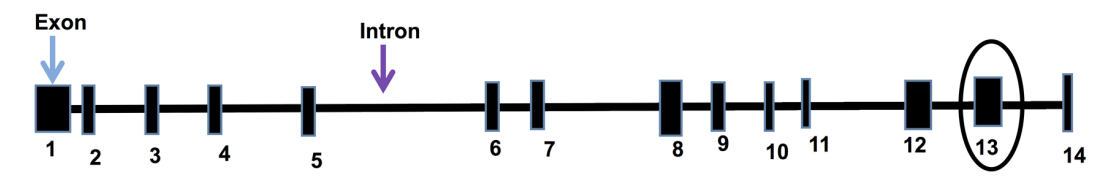

$\stackrel{\text { MePDSF }}{\longrightarrow}$

B ...atccacacccattaggccttgtaatgaacccccaaaagtttgttttgtccttttattgtctgtgtctgcatgtgcatgtgcaatttttcaccttggctaaaccaagttt aagcatcaagtacCTGTACTATTGACTGCGTACAAAGCTTCCCAGATAGGACAGCGCCCTCCATTGAAGCCAAAT ATTTTTGCTTTGTGTAGTCCCCAGCTAAATAGAAGCCCTCTATAGGAGATCTCTGTAAGGGACGGCAAGGTT CACAATTTGGAACCGTCTTGTAAACAGATctgagaaaatcaccaggtaaccagaagggaaatgactaacgagattagtgatcaatatcttt caatggttgaattttccatatgaagatacaaggagagaaaaaataagtgcaatataatgttagcagaagcatatgaagtgctatgtagtaaacacaaaaca aatattgatagagctgtattagcaagaaattcaattgaatggctttactctcc... MePDS R

Construct gRNA gRNA Sequence

MePDS-1 gRNA1 GCGTACAAAGCTTCCCAGAT

MePDS-2 gRNA2 GATCTCTGTAAGGGACGGCA

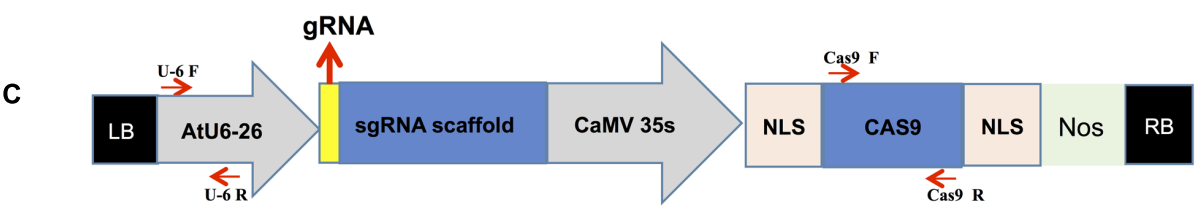

FIGURE 1 | Schematic representations of the cassava MePDS target gene, location of the gRNAs, and CRISPR/Cas9 gene-editing construct. (A) Structural organization of the MePDS gene. Exons and introns are shown as boxes and lines, respectively, with the number of exons indicated below boxes. The gRNA where designed from the encircled exon 13. (B) Schematic representation of target region showing the sequences and location of the two 20 bp gRNAs. The sequence shown is a reverse complement of MePDS from cultivar AM560-2 (Phytozome 11, cassava4.1_004359m.g). gRNA1 and gRNA2 are highlighted in yellow and green, respectively, within exon 13 shown as upper case and surrounding intron sequences appears as lower case. Positions of forward (F) and reverse (R) primers flanking the target region in MePDS are indicted with red arrows, respectively. (C) Schematic of the CRISPR/Cas9 binary vector pCAMBIA2300_CR3-EF used for stable Agrobacterium-mediated transformation of cassava. Arabidopsis thaliana promoter (AtU6-26) drives expression of each gRNA used to target MePDS, with the gRNA ligated at the position indicated by the yellow box aided by the Bsa1 restriction site. The Cauliflower mosaic virus promoter (CaMV $35 S$ ) drives expression of the Cas9 gene which together with inserted gRNA induce mutations in target region of MePDS gene; NLS, nuclear localization signal; Nos, Nos terminator; LB, left border; RB, right border. Position of forward $(F)$ and reverse $(R)$ primers used for amplifying respective regions of cassette are shown by red arrows.

2015). Regenerated cotyledon-stage somatic embryos and whole plantlets with altered PDS activities were visually discriminated from control tissues under a dissection microscope. Plants were established in the greenhouse by transfer to Fafard 51 soilless potting medium (Conrad Fafard, Inc., Agawam, MA, United States) before moving them to the open bench and grown at a temperature of $26^{\circ} \mathrm{C} / 25^{\circ} \mathrm{C}$ (day/night) with $60-90 \%$ relative humidity for 3 weeks to reach approximately $8 \mathrm{~cm}$ in height, at which time they were transferred to a glasshouse maintained at a temperature of $32^{\circ} \mathrm{C} / 27^{\circ} \mathrm{C}$ (day/night) and $70-95 \%$ relative humidity (Taylor et al., 2012).

\section{Detection of CRISPR/Cas9 Induced Mutations}

Leaf tissue was collected from rooted in vitro plantlets. Approximately $0.1 \mathrm{~g}$ of leaf tissue was placed in a $2-\mathrm{ml}$ screw cap tube containing ceramic beads and crushed to fine powder using a Fast-Prep 24 (MP Biomedicals, Solon, OH, United States) set at $4.0 \mathrm{~m} / \mathrm{s}$ for $30 \mathrm{~s}$ twice. Genomic DNA was extracted using cetyltrimethylammonium bromide $(\mathrm{CTAB})$ protocol (Doyle and Doyle, 1990) and treated with RNase, DNase-free kit (Roche Diagnostics $\mathrm{GmbH}$, Indianapolis, IN, United States) to remove RNA.
Putatively edited plant lines were subjected to polymerase chain reaction (PCR) to confirm integration of the T-DNA using $200 \mathrm{ng}$ genomic DNA as a template and primers specific for the Cas9 gene, U6-26 promoter, and gRNA scaffold (Table 1), under the following conditions: $98^{\circ} \mathrm{C}$ for $5 \mathrm{~min}$; 34 cycles of $98^{\circ} \mathrm{C}$ for $10 \mathrm{~s}, 65^{\circ} \mathrm{C}$ for $20 \mathrm{~s}, 72^{\circ} \mathrm{C}$ for $30 \mathrm{~s}$ followed by final extension of $72^{\circ} \mathrm{C}$ for $5 \mathrm{~min}$. Genomic DNA template was used for PCR amplification of a 504-bp fragment spanning the targeted MePDS sequence. Briefly, 200 ng of DNA was added to Phusion DNA Polymerase Master Mix (New England Biolabs ${ }^{4}$ ) in a $25 \mu \mathrm{l}$ reaction volume and $\mathrm{PCR}$ performed at $98^{\circ} \mathrm{C}$ for $5 \mathrm{~min} ; 34$ cycles of $98^{\circ} \mathrm{C}$ for $10 \mathrm{~s}, 64^{\circ} \mathrm{C}$ for $20 \mathrm{~s}, 72^{\circ} \mathrm{C}$ for $30 \mathrm{~s}$, followed by final extension at $72^{\circ} \mathrm{C}$ for $5 \mathrm{~min}$. A single primer pair was designed to amplify both target sites based on the MePDS gene sequence from reference genotype AM560-2 in Phytozome 11, version 4.1. PCR products were resolved on a $1.5 \%$ agarose gel, excised, and amplicons purified using the QIAquick gel extraction kit (Qiagen Group), before cloning into pCR BluntII TOPO vector (Life Technologies ${ }^{5}$ ). Between 5 and 10 single colonies per plant line were subjected to Sanger sequencing

\footnotetext{
${ }^{4}$ https://www.neb.com/

${ }^{5}$ https://www.thermofisher.com/us/en/home.html
} 
TABLE 1 | Primers used to confirm integration of T-DNA and integrity of MePDS target sequence.

\begin{tabular}{|c|c|c|c|}
\hline Primer & Forward sequence & Reverse sequence & Product size (bp) \\
\hline MePDS & GGAGAGTAAAGCCATTCAATTG & ATCCACACCCATTAGGCCTTG & 504 \\
\hline Cas9 Gene & GCTGGGCCGTGATCACCGAC & САCTCTCAGGATGTCGCTCAGC & 900 \\
\hline U6 Promoter & CAGGAAACAGCTATGACCATG & CATGTTGACCTGCAGGCA & 700 \\
\hline
\end{tabular}

TABLE 2 | Production and recovery of albino plants after CRISPR/Cas9-mediated gene editing of MePDS.

\begin{tabular}{|c|c|c|c|c|c|c|c|c|c|c|}
\hline \multirow[b]{2}{*}{ Construct } & \multicolumn{5}{|c|}{60444} & \multicolumn{5}{|c|}{ TME 204} \\
\hline & $\begin{array}{c}\text { Somatic } \\
\text { embryo } \\
\text { lines }\end{array}$ & $\begin{array}{l}\text { Plant lines } \\
\text { recovered }\end{array}$ & $\begin{array}{l}\text { Fully } \\
\text { albino } \\
\text { lines }\end{array}$ & $\begin{array}{c}\text { Partially } \\
\text { albino } \\
\text { lines }\end{array}$ & $\begin{array}{l}\text { Fully } \\
\text { green } \\
\text { lines }\end{array}$ & $\begin{array}{c}\text { Somatic } \\
\text { embryo } \\
\text { lines }\end{array}$ & $\begin{array}{l}\text { Plant lines } \\
\text { recovered }\end{array}$ & $\begin{array}{c}\text { Fully } \\
\text { albino } \\
\text { lines }\end{array}$ & $\begin{array}{c}\text { Partially } \\
\text { albino } \\
\text { lines }\end{array}$ & $\begin{array}{c}\text { Fully } \\
\text { green } \\
\text { lines }\end{array}$ \\
\hline MePDS-1 & 45 & 23 & 2 & 19 & 2 & 38 & 9 & 7 & 2 & 0 \\
\hline MePDS-2 & 58 & 27 & 10 & 16 & 1 & 47 & 10 & 6 & 3 & 1 \\
\hline EV control & 11 & 5 & 0 & 0 & 5 & 20 & 4 & 0 & 0 & 4 \\
\hline WT & 8 & 3 & 0 & 0 & 3 & 9 & 2 & 0 & 0 & 2 \\
\hline Total & 122 & 58 & 12 & 35 & 11 & 114 & 25 & 13 & 5 & 7 \\
\hline
\end{tabular}

EV, empty vector control; WT, non-transgenic wild-type control.

using M13F and M13R primers to characterize CRISPR/Cas9 induced mutations. Sequences were aligned with the wild-type reference sequence of $M e P D S$ gene using the MUSCLE alignment program of EMBL-EBI ${ }^{6}$. The mutation frequency in transgenic plants was calculated by expression of plant lines with phenotypic alterations or mutated sequences as a percentage of total plant lines examined.

\section{RESULTS}

The nucleotide sequence of $A$. thaliana PDS (AtPDS: NM117498) was used as bait to search for homologous sequences within the reference cassava genome in Phytozome 11 (version 4.1) and a single copy of $M e P D S$ identified. The genomic sequence of $M e P D S$ is 13,130 nucleotides in size, comprising 14 exons and 13 introns (Figure 1A), with 1,743 nucleotides of transcript sequence coding for 580 amino acids. AtPDS (NP_193157.1) and MePDS (Manes.05G193700.1) are 85\% identical at the protein level, suggesting a conserved function in cassava. In order to disrupt $M e P D S$, two distinct gRNAs were designed as predicted by the CRISPR-P program to have the highest efficacy and least off-target potential. The gRNAs were cloned into two separate binary vectors (MePDS-1 and MePDS-2) carrying the Cas9 gene (Figure 1B), Arabidopsis U6-26 promoter and gRNA scaffold (Figure 1C), and delivered by Agrobacterium into embryogenic cells of cassava.

\section{PDS Edited Plants Exhibit Dwarf and Albino Phenotypes}

A total of 103 independent lines of regenerating somatic embryos were recovered from cv. 60444 and 85 from cv. TME 204 after selection on antibiotic-containing medium (Chauhan et al.,

${ }^{6} \mathrm{http}: / /$ www.ebi.ac.uk/Tools/msa/muscle/
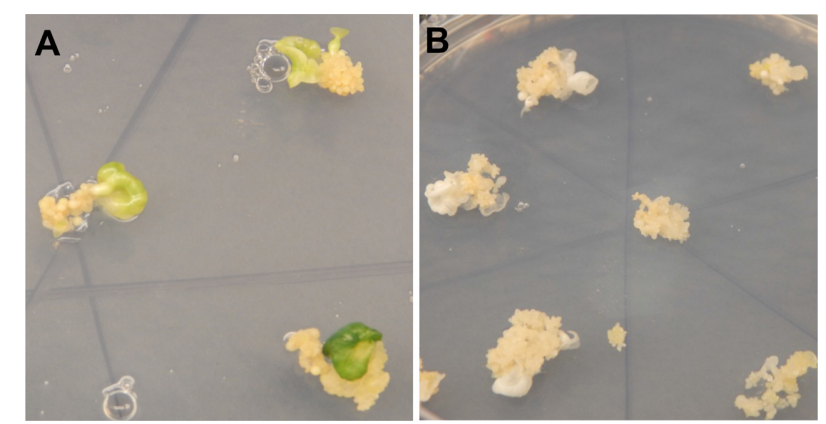

FIGURE 2 | Regenerating cassava somatic embryos after CRISPR/Cas9-mediated mutation of MePDS. (A) Fully green cotyledon stage embryos regenerating from non-edited wild-type tissues of cv. 60444. (B) Regenerating albino cotyledons with CRISPR/Cas9 edited MePDS.

2015) (Table 2). Visual indication of altered MePDS function was first apparent as albino or partial albino cotyledon-stage somatic embryos regenerating on selection medium (Figure 2). This phenotype was observed in 100/103 (97.1\%) and 84/85 (98.9\%) somatic lines of cv. 60444 and TME 204 transgenic for $M e P D S-1$ and $M e P D S-2$, respectively. Both constructs were equally effective for producing the mutated phenotype. A total of $47 \%$ of the somatic embryo lines from cv. 60444 and $22 \%$ from $\mathrm{cv}$. TME 204 germinated to produce plantlets, generating 58 and 25 independent transgenic plant lines from 60444 and TME 204, respectively.

A diversity of albino phenotypes was observed in the regenerated plants, ranging from full albinos with total loss of green coloration, to partial albinos displaying a mixture of green and white tissues on the same plantlet (Figures 2, 4). In all cases, non-edited wild-type, EV and GFP-expressing control plants remained fully green. Both gRNAs generated albino mutants with high frequencies ranging from $90 \%$ for $M e P D S-2$ to 
A

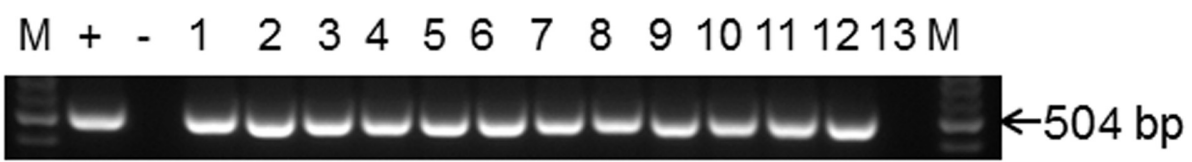

B

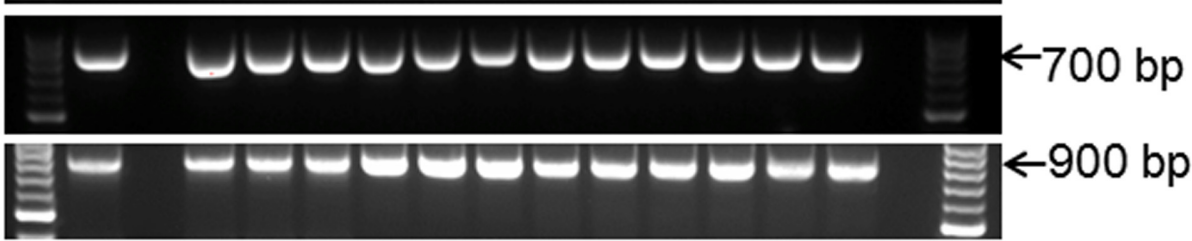

FIGURE 3 | PCR amplification and detection of CRISPR/Cas9 T-DNA integration and the MePDS target sequence. (A) PCR amplification for 504 bp of the MePDS target region (B) confirmation of CRISPR/Cas9 presence by detection of the Cas9 gene. (C) The U6-26 promoter and gRNA scaffold. The expected band sizes are shown on the left as 504, 900, and 700 bp for (A-C), respectively. M is molecular size marker. For (A), + is an edited positive reference sample from 60444 confirmed by sequencing, lanes 1-11 display PCR amplicons from MePDS edited plant lines lanes, lane 12 is a wild-type plant, and lane 13 is water control. For $(\mathbf{B}, \mathbf{C})+$ is plasmid DNA containing the binary vector pCAMBIA2300, - is a no template control, lanes 1-12 are PCR products from selected MePDS edited plant lines, and lane 13 a wild-type control.

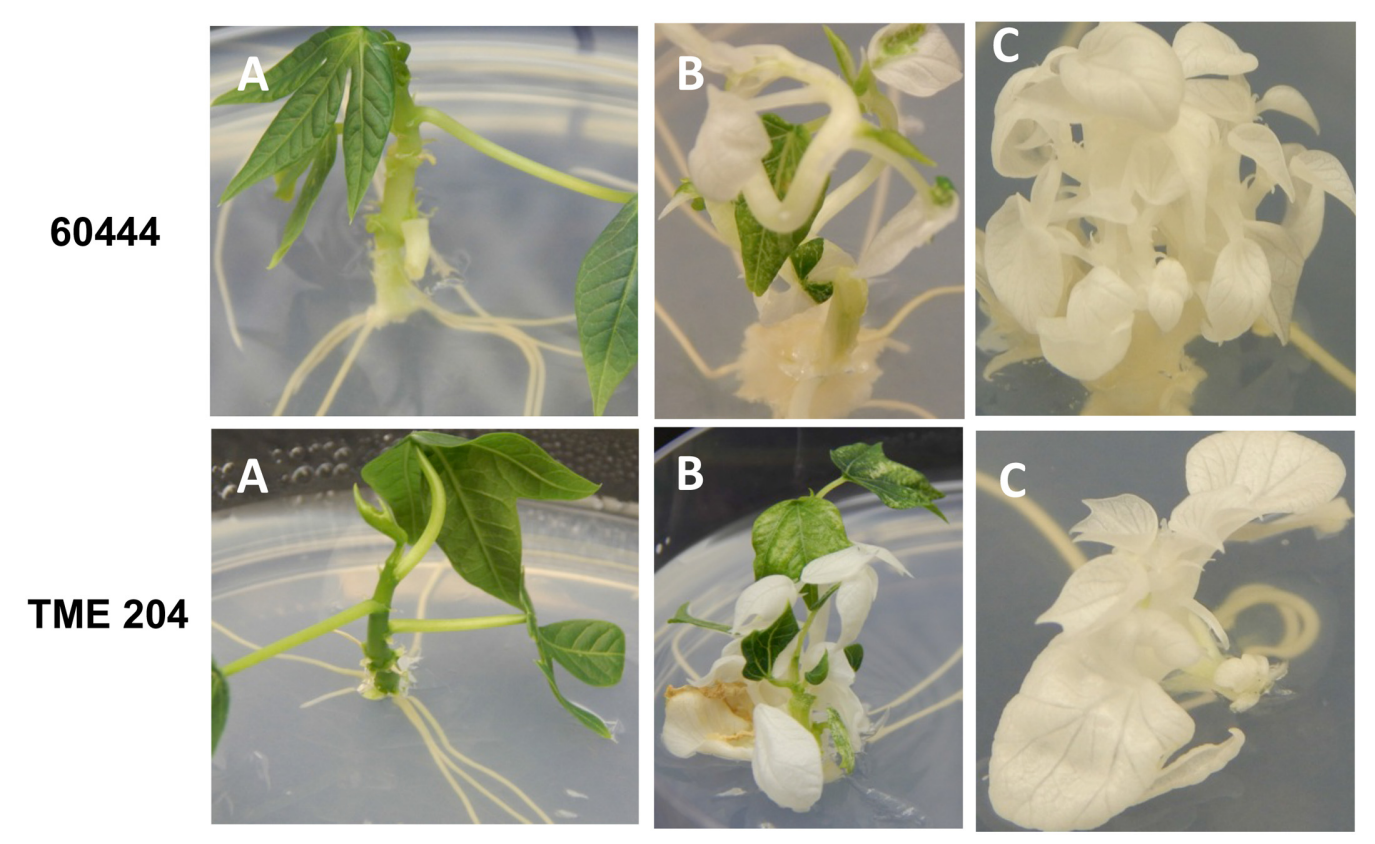

FIGURE 4 | Phenotypic diversity of CRISPR/Cas9 induced MePDS mutations in in vitro cassava plantlets. Upper panel shows cv. 60444, and lower panel, cv. TME 204. (A) Non-edited wild-type control plants with fully green shoots, (B) chimeric albino plant showing a mixture of green and white shoot tissues, and (C) fully albino plants.

100\% for MePDS-1 in cv. TME 204 (Figure 6A). Mutant plants characterized by full or partial albino phenotypes displayed diminished growth rates, shorter petioles, and reduced leaf area compared to the non-edited control equivalents (Figures 4, 5). Albino plant lines with greatly or totally diminished green tissue (Figures 4, 5A) did not survive more than two to three successive in vitro propagation cycles. A selected set of partial albino and control plants was transferred to soil, where the dwarf phenotype and albino nature were maintained when grown under greenhouse conditions. In contrast, control EV and GFP-expressing control plants were fully green and showed robust growth (Figure 5).

\section{Molecular Analysis of CRISPR/Cas9 Induced Mutations in MePDS Gene}

Polymerase chain reaction (PCR) was performed on genomic DNA extracted from regenerated plants to detect the presence of the T-DNA and to amplify the targeted MePDS gene (Table 1). As expected, all albino and EV plant lines tested positive for presence of the Cas9 gene, U6-26 promoter, and gRNA scaffold (Figures 3B,C). A 504 bp fragment corresponding to the target region of the MePDS gene in cassava was amplified (Figure 3A), and amplicons from 38 plant lines (19 each for 60444 and TME 204) were cloned and 241 clones sequenced (Table 3). All full or partial albino plants were found to carry 

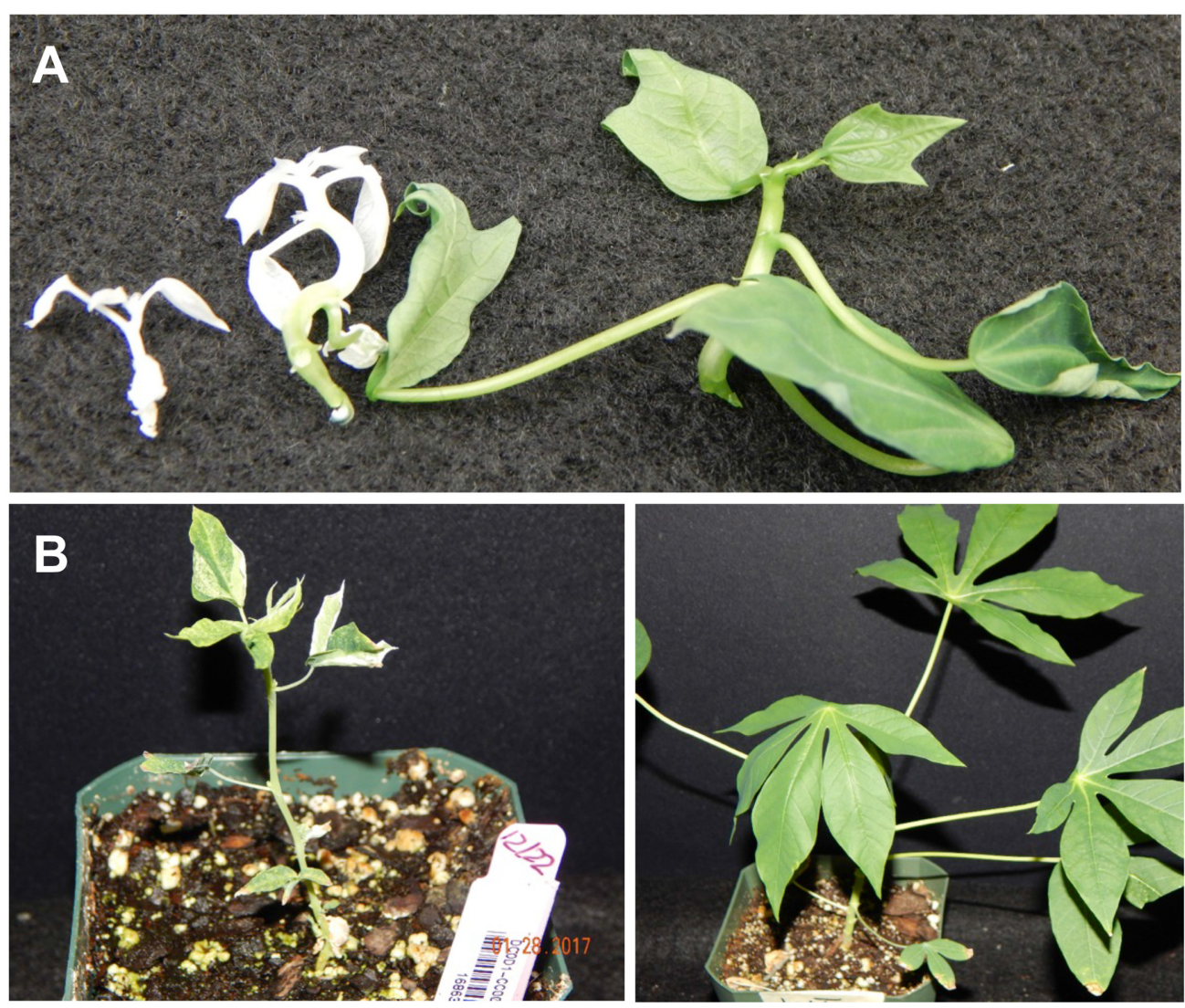

FIGURE 5 | Effects of CRISPR/Cas9 directed alteration of MePDS gene function on phenotypes of regenerated cassava plants. (A) Dwarf in vitro plants of cv. TME 204 edited with MePDS-2 with green non-modified plant on the right, (B) greenhouse grown plants of cv. 60444 edited with MePDS-1 characterized by stunted growth, shorter petioles, and reduced leaf area compared to the robust fully green non-edited wild-type control on the right.

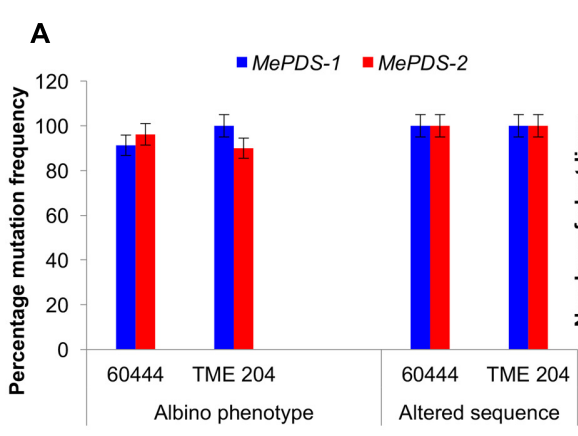

Plant lines

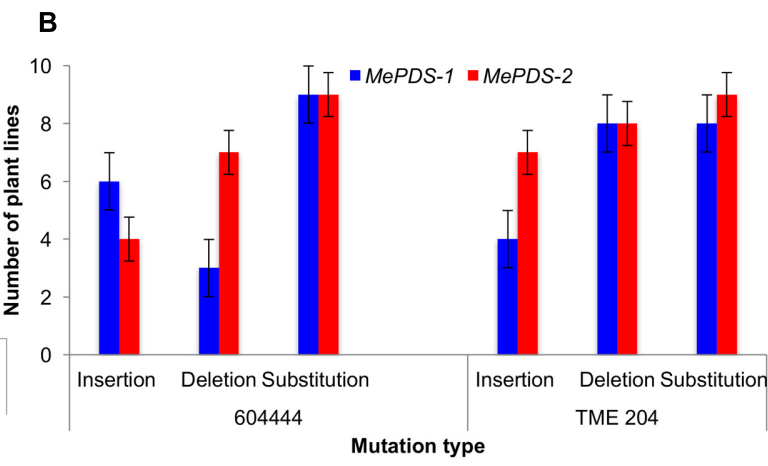

FIGURE 6 | CRISPR/Cas9 induced mutation frequency and types. (A) Percentage frequency of mutation based on occurrence of albino phenotypes and recovered altered sequence in plant lines of cv. 60444 and cv. TME 204. Albino mutation frequency was calculated as number of germinated albino plants lines expressed as percentage of total number of plant lines generated. Frequency of plants with altered sequence was obtained by expressing number of plant lines with sequence alteration as a ratio of total plant lines sequenced. (B) Number of plant lines with the various induced mutations following alignment of wild-type sequence with the test sequences. MePDS-1 and MePDS-2 are plasmids carrying the gRNAs used to target MePDS gene.

mutations in their MePDS gene, with the MePDS-1 and MePDS-2 constructs equally efficacious for inducing mutations in the two cultivars tested (Table 3). Both INDELs and substitutions were observed. Regardless of the construct and cultivar tested, substitutions occurred more frequently than insertions and deletions (Figure 6B). Constructs MePDS-1 and MePDS-2 directed insertion or substitution of different nucleotides. In cv. 60444, both constructs induced insertion and substitution 


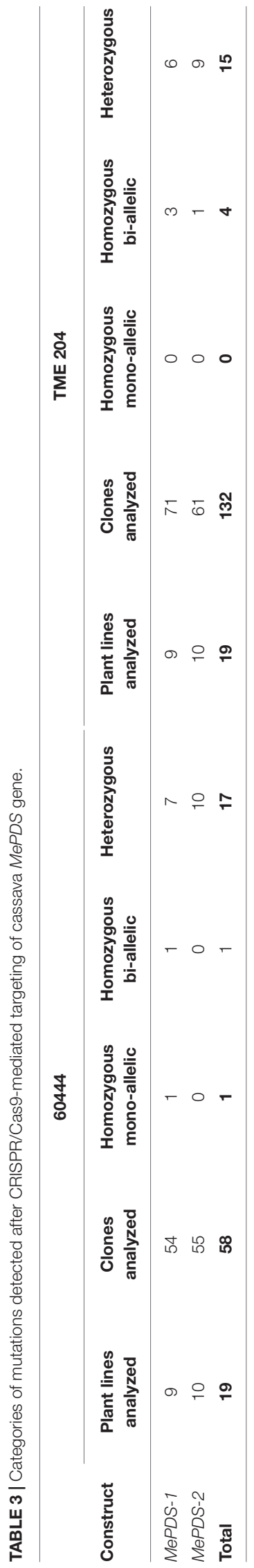

of nucleotide ' $\mathrm{A}$ ' compared to substitution of nucleotides ' $\mathrm{CA}$ ' and ' $\mathrm{T}$ ' in $\mathrm{cv}$. TME 204. The longest deletion detected in $\mathrm{cv}$. 60444 was $16 \mathrm{bp}$, compared to $101 \mathrm{bp}$ observed in cv. TME 204 plant line 4 (Supplementary Figure S1). Examination of the 504 bp MePDS target sequence after Sanger sequencing revealed $1 \mathrm{bp}$ nucleotide substitutions and INDELs upstream of the $5^{\prime}$ and or downstream of the $3^{\prime} M e P D S$ target region for both constructs MePDS-1 and MePDS-2. We observed a single nucleotide polymorphism (SNP), which overlapped with MePDS2 gRNA ('C' substituted for ' $G$ ') targeting the MePDS region of cv. TME 204, but this did not negatively affect CRISPR/Cas9 editing of MePDS.

The approach developed by Fan et al. (2015) in populus was used to classify plant lines as putatively homozygous or heterozygous for the induced mutations with slight modification. Sequences derived from albino mutants were aligned and compared with the reference wild-type sequence of MePDS. Mutant lines in which all clones examined carried one identical type of mutation were classified as putatively homozygous monoallelic, while mutants whose clones displayed two identical types or patterns of mutations were considered to be putatively homozygous bi-allelic. Mutants categorized as heterozygous were chimeric, having at least three types or patterns of mutations and/or wild-type sequence present in the same plant. Based on this scheme, 1/19 plant lines (5.23\%, line 9) of cv. 60444 was putatively homozygous mono-allelic since all five clones carried two substitutions (Figure 7). Further scrutiny revealed that $1 / 19$ plant lines $(5.23 \%$, line 8 ) of cv. 60444 and $4 / 19$ plant lines (21.1\%, lines 2, 6, 7, and 9) of cv. TME 204 were putatively homozygous bi-allelic (Table 3); 17/19 (89.5\%) of cv. 60444 and 15/19 (78.9\%) of cv. TME 204 mutant plant lines were categorized as heterozygous (Table 3). Assessment of sequence and phenotypic data further indicated that the majority of full albino plant lines edited with MePDS-1 were putatively homozygous for mutations at MePDS (Table 3). Plant line 5 of cv. TME 204 which remained fully green showed sequence alteration in only one of the two MePDS alleles (Figure 7). The altered sequence (putatively homozygous or heterozygous) and albino (full or partial albino) frequency data agree with over $90 \%$ of CRISPR/Cas9 directed mutation frequency (Figure 6A). When the $504 \mathrm{bp} M e P D S$ target sequence was examined for the presence of off targets, Sanger sequencing revealed $1 \mathrm{bp}$ nucleotide substitutions and INDELs upstream of the $5^{\prime}$ and or downstream of the $3^{\prime}$ target region. In most cases, such off-target effects occurred next to a random PAM site NGG or NCC within the MePDS gene.

\section{DISCUSSION}

CRISPR/Cas9 genome-editing technology has become an effective tool for basic research and trait development in crop plants (Pennisi, 2013; Zhang et al., 2017). To date, however, no such capacity has been reported for the tropical crop cassava. Efficient gene editing in this important staple food crop would present new opportunities to address biotic 


\section{MePDS-1, cv. 60444}

WT ACTGCGTACAAAGCTTCCC-AGATAGGACAGCGCCCTCCATTGAAGCCAAATATTTTTGCTTTGTGTAGTCCCCA

-3(1) ACTGCGTACAAAGCTTCCC-AGATAGGACAGCGCCCTCCATTGAAGCCAAATATTTTTGCTTTGTGTAGTCCCCA 0

(4) ACTGCGTACAAAGCTTCCCAAGATAGGACAGCGCCCTCCATTGAAGCCAAATATTTTTGCTTTGTGTAGTCCCCA +1

(1) ACTGCGTACAA------AGATAGGACAGCGCCCTCCATTGAAGCCAAATATTTTTGCTTTGTGTAGTCCCCA -8

(1) ACTGC----.----ATAGGACAGCGCCCTCCATTGAAGCCAAATATTTTTGCTTTGTGTAGTCCCCA -16 $* * * * * \quad * * * * * * * * * * * * * * * * * * * * * * * * * * * * * * * * * * * * * * * * * * * * * * * * * * * *$

WT ACTGCGTACAAAGCTTCCC-AGATAGGACAGCGCCCTCCATTGAAGCCAAATATTTTTGCTTTGTGTAGTCCCCA

-8 (4) ACTGCGTACAAAGCTTCCCAAGATAGGACCGCGCCCTCCATTGAAGCCAAATATTTTTGCTTTGTGTAGTCCCCA $* * * * * \quad * * * * * * * * * * * * * * * * * * * * * * * * * * * * * * * * * * * * * * * * * * * * * * * * * * *$

WT GCTAAATAGAAGCCCTCTATAGGAGATCTCTGTAAGGGACGGCAAGGTTCGCAATTTGGAACCGTCTTGTAAACA

-8(4) GCTAAATAGAAGCCCTCTATAGGAGATCTCTGTAAGGGACGGCAAGGTTCACAATTTGGAACCGTCTTGTAAACA

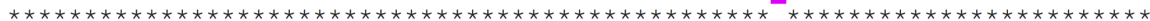

WT GATCTGAGAAAATCACCAGGTAACCAGAGGGGAAATGACTAACGAGATTAGTGATCAATATCTTTCAATGGTTGA

-8(4) GATCTGAGAAAATCACCAGGTAACCAGAGGGAAATGACTAACGAGATTAGTGATCAATATCTTTCAATGGTTGA +1, s2

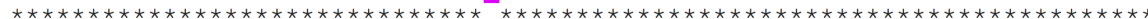

WT ACTGCGTACAAAGCTTCCCAGATAGGACAGCGCCCTCCATTGAAGCCAAATATTTTTGCTTTGTGTAGTCCCCAG

-9(5) ACTGCGTACAAAGCTTCCCAGATAGGACAGCGCCCTCCATTGAAGCCAAATATTTTTGCTTTGTGTAGTCCCCAG

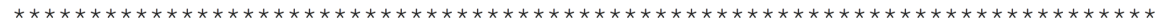

WT CTAAATAGAAGCCCTCTATAGGAGATCTCTGTAAGGGACGGCAAGGTTCGCAATTTGGAACCGTCTTGTAAACAG

-9(5) CTAAATAGAAGCCCTCTATAGGAGATCTCTGTAAGGGACGGCAAGGTTCACAATTTGGAACCGTCTTGTAAACAG

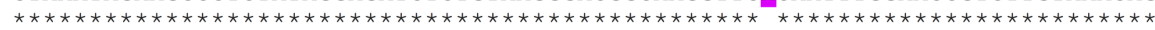

WT ATCTGAGAAAATCACCAGGTAACCAGAGGGGAAATGACTAACGAGATTAGTGATCAATATCTTTCAATGGTTGAA

-9(5) ATCTGAGAAAATCACCAGGTAACCAGAGGGAAATGACTAACGAGATTAGTGATCAATATCTTTCAATGGTtGAA S2

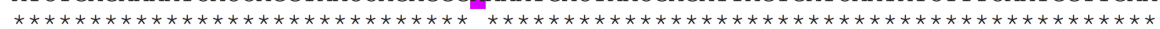

MePDS-1, CV. TME 204

WT TTGGCTAAACCAAGTTTAAGCATCAAGTACCTGTAATATTGACTGCGTACAAAGCTTCCCAGATAGGACAGCGCC

-6(4) TTGGCTAAACCAAGTTTAAGCATCAAGTACCTGTACAATTGACTGCGTACAAAGCTTCCC-GATAGGACAGCGCC -1

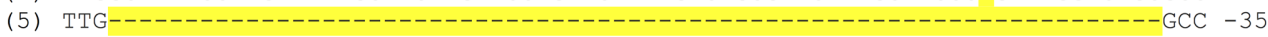

WT GCGTACAAAGCTTCCC-AGATAGGACAGCGCCCTCCATTGAAGCCAAATATTTTTGCTTTGTGTAGTCCCCAGCT

-9 (9) GCGTACAAAGCTTCCCAAGATAGGACAGCGCCCTCCATTGAAGCCAAATATTTTTGCTTTGTGTAGTCCCCAGCT **************************************************************************

WT AAATAGAAGCCCTCTATAGGAGATCTCTGTAACGGACGGCAAGGTTCACAATTTGGAACCGTCTTGTAAACAGAC

-9(9) AAATAGAAGCCCTCTATAGGAGATCTCTGTAACGGACGGCAAGGTTCACAATTTGGAACTGTCTTGTAAACAGAC +1, S1

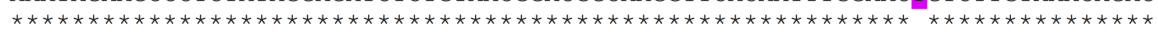

MePDS-2, cv. 60444

WT GGAGATCTCTGTAAgGGAC-GgCAAgGTTCACAATTTGGAACCGTCTTGTAAACAGATCTGAGAAAATCACCAG

-6 (1) GGAGATCTCTGTAAG----GGCAAGTTCACAATTTGGAACCGTCTTGTAAACAGATCTGAGAAAATCACCAG -4

(2) GGAGATCTCTGTAA------GCAAGGTTCGCAATTTGGAACCGTCTTGTAAACAGATCTGAGAAAATCACCAG -6, S1

(1) GGAGATCTCT----_---_GCAAGGTTCGCAATTTGGAACCGTCTTGTAAACAGATCTGAGAAAATCACCAG -10, S1

(3) GgAGATCTCTGTAAgGGACGgGCAAGgTTCACAATTTGGAACCGTCTTGTAAACAGATCTGAGAAAATCACCAG +1

-7 (3) GGAGATCTCTGTAAGGGAC-GGCAAGGTTCACAATTTGGAACCGTCTTGTAAACAGATCTGAGAAAATCACCAG 0

(1) GgAGATCTCTGTAAGGGAC-GGCAAgGTTCGCAATTTGGAACCGTCTTGTAAACAGATCTGAGAAAATCACCAG S1

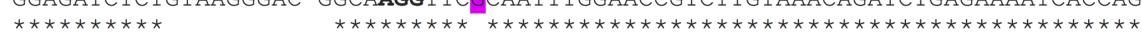

MePDS-2, Cv. TME 204

WT GGAGATCTCTGTAACGGACG-GCAAGgTTCACAATTTGGAACCGTCTTGTAAACAGACCTGAGAAAAATCAGCA

-3(1) GGAGATCTCTGTAACGGACGGGCAAGGTTCACAATTTGGAACCGTCTTGTAAACAGACCTGAGAAAAATCAGCA +1

(3) GGAGATCTCTGTAACGGACGGGCAAGgTTCACAATTTGGAACTGTCTTGTAAACAGACCTGAGAAAAATCAGCA +1, S1

(2) GGAGAT-------------AAGGTTCACAATTTGGAACCGTCTTGTAAACAGACCTGAGAAAAATCAGCA -16

-4(3) GGAGATCTCTGTAACGGACG-GCAAGGTTCACAATTTGGAACCGTCTTGTAAACAGACCTGAGAAAAATCAGCA

(1) GGAGATCTCTGTAACGGACGCGCAAggTTCACAATTTGGAACCGTCTTGTAAACAGACCTGAGAAAAATCAGCA +1

(2) GgAGATCTCTGTAACGGACGCGCAAGgTTCACAATTTGGAACTGTCTTGTAAACAGACCTGAGAAAAATCAGCA +1, S1

-5(1) GGAGATCTCTGTAACGGACG-GCAAGgTTCACAATTTGGAACCGTCTTGTAAACAGACCTGAGAAAAATCAGCA 0

(6) GGAGATCTCTGTAACGGACG-GCAAGGTTCACAATTTGGAACTGTCTTGTAAACAGACCTGAGAAAAATCAGCA S1

-8 (4) GGAGATCTCTGTAACGGAC--GCAAGGTTCACAATTTGGAACCGTCTTGTAAACAGACCTGAGAAAAATCAGCA -1

(1) GGAGATCTCTGTAACGGAC--GCAAgGTTCACAATTTGGAACTGTCTTGTAAACAGACCTGAGAAAAATCAGCA -1, S1 $\star \star \star \star \star * \star$

FIGURE 7 | Sequence-based detection of mutations induced by CRISPR/Cas9 constructs MePDS-1 and MePDS-2 in cultivars 60444 and TME 204 . For each plant line characterized, PCR was used to amplify across the target region. The PCR product was cloned into pCR Blunt-II TOPO vector and transformed into E. coli. Multiple individual colonies were analyzed via Sanger sequencing to detect mutations near the target site. Aligned sequence data is shown for 11 representative mutant plant lines. The target region of MePDS is underlined and bolded in the wild-type (WT) reference sequence, with the protospacer adjacent motif (PAM) in bold but not underlined. Sequences from each plant line $(-3,-8$, etc.,) are shown below the WT sequence. The number of colonies with a specific sequence pattern is indicated in parentheses. Deletions are highlighted in yellow, insertions are highlighted green, and substitutions are highlighted in pink. Mutation type insertion (+), deletion (-), or substitution (S) and size are indicated at the right side of the panel.

and abiotic constraints in cassava production and postharvest utilization (Bart and Taylor, 2017). Toward this end, CRISPR/Cas9 was employed to target the PDS gene in cassava.
PDS codes for key enzymes in the carotenoid biosynthesis pathway (Qin et al., 2007). Defects in PDS gene function cause dwarfism and albino phenotypes in Arabidopsis due to impaired 
chlorophyll, carotenoid, and gibberellin biosynthesis (Qin et al., 2007). Reports of CRISPR/Cas9 targeting of PDS in rice, apple, sweet orange, and populus resulted in similar phenotypic aberrations (Jia and Wang, 2014; Zhang et al., 2014; Fan et al., 2015; Nishitani et al., 2016). Deliberate alteration of the PDS gene was therefore chosen as a visual marker to reveal efficacious application of CRISPR/Cas9-targeted genome editing in cassava. PDS offers an attractive target for developing gene-editing techniques due to its monogenic nature and the easily assessed albino phenotype resulting from its mutation (Jia and Wang, 2014; Wang et al., 2015; Nishitani et al., 2016).

In order to maximize our chance of success, two gRNAs were designed to interrupt MePDS function by targeting exon 13 in cassava cultivars 60444 and TME 204. The $13^{\text {th }}$ exon was chosen because CRISPR-P output indicated that it provided optimal gRNAs starting with ' $G$ ' for use with the U6 promoter for achieving successful gene editing. In addition, previous studies report that mutations to the PDS gene at any location translate into phenotypic alteration. Existing genetic transformation systems (Taylor et al., 2012; Chauhan et al., 2015) were employed to integrate the CRISPR/Cas 9 tools into embryogenic cells. Albino phenotypes were recovered from both cultivars after transformation with two gRNA constructs (Figures 2, 4, 5 and Table 2). Albino somatic embryos were visualized as early as 8 weeks after transformation and could be regenerated to plantlets displaying full and partial albino characteristics (Figures 2, 4, 5), in a manner similar to that reported in other plant species (Qin et al., 2007; Jia and Wang, 2014; Fan et al., 2015; Nishitani et al., 2016). Recovery of the albino mutants occurred at unexpectedly high frequencies of 93-95\% of the regenerated plant lines of cvs. 60444 and TME 204, respectively. The albino phenotypes observed in the current study resulted from loss of MePDS function generated by action of the CRISPR/Cas9 reagents. The observed diversity and degree of the albino phenotype recorded here could have resulted from differential timing of the targeted mutation. Due to the integrated nature of the editing tools, editing will continue from CRISPR/Cas9 expressing cassettes within a given cell until the target site is mutated. This can result in chimeric plants consisting of cells with non-modified genomes, and populations of cells carrying different gene edited events. The exact pattern of these would depend on timing of CRISPR/Cas9-mediated editing in relation to the plant regeneration process. In rice, for example, the occurrence of full albino mutants totally lacking chlorophyll and partial albinos characterized by a mixture of albino and green tissues was attributed to induction of mutations before and after first cell division (Zhang et al., 2014).

Molecular analysis was performed on albino and green plant lines, with multiple clones sequenced per plant line to identify mutations within one or both MePDS alleles. Sequence data indicated the presence of INDELs and substitutions in all (100\%) of the 38 (19 from each cultivar) regenerated plant lines examined at the DNA level. Irrespective of cultivar and construct used, INDELs mostly occurred 1-3 bp upstream from the PAM site. Predominantly single nucleotide insertions and substitutions (Figure 7) were detected in MePDS resulting from both gRNA constructs. However, larger deletions, including 1 of $101 \mathrm{bp}$ in TME 204, were also observed (Supplementary Figure S1). The INDELs recorded here could have resulted from NHEJ repair following integration of our constructs $M e P D S-1$ and $M e P D S-2$ as previously reported in apple and populus following CRISPR/Cas9-targeted mutation of the PDS gene (Fan et al., 2015; Nishitani et al., 2016). Observation of single nucleotide substitutions upstream or downstream of the target region (Figures 6B, 7 and Supplementary Figure S1) implies activation of HR pathway to repair DSBs created in $M e P D S$. Simultaneous triggering of $\mathrm{HR}$ and NHEJ repair pathways proceeding integration of CRISPR/Cas9 reagents was documented earlier in apple, $N$. benthamiana, and cotton (Li et al., 2013; Nishitani et al., 2016; Gao et al., 2017). We also recovered wild-type sequences from some colonies derived from plants that clearly showed altered phenotype (Figure 7). This finding is similar to data reported for CRISPR/Cas9 gene targeting in N. benthamiana and rice (Nekrasov et al., 2013; Zhang et al., 2014). Gene edited TME 204 plant line \#4, transgenic for $M e P D S-1$, returned three clones with double nucleotide substitutions (CA) (Supplementary Figure S1). This rare occurrence seems to indicate that CRISPR/Cas9 can direct differing frequency and types of mutations. Induction of high mutation rates by $M e P D S-2$ gRNA that carried a single bp mismatch with the cv. TME 204 target sequence was common. This finding is not surprising in light of a similar recent report in which a target sequence with a single nucleotide difference was deliberately chosen to successfully induce CRISPR/Cas9 directed mutation in homologous Chloroplastos alterados 1 genes of cotton (GhCLA1) (Gao et al., 2017). Four transgenic plant lines out of the $69(4 / 69,5.8 \%)$ examined displayed unexpected visible green phenotype like WT and EV controls. However, sequence data showed presence of nucleotide substitutions in one allele of $M e P D S$ in these plants (Figure 7). This phenomenon is consistent with results obtained in citrus, Arabidopsis, and N. benthamiana (Li et al., 2013; Nekrasov et al., 2013; Shan et al., 2013; Jia and Wang, 2014), and could result from presence of very low populations of photo-bleached PDS cells among non-modified tissues (Li et al., 2013). Alternatively, the presence of a single, fully functional allele may be sufficient to ensure functionality of MePDS. Possibly because of the high mutation efficiency achieved, we simultaneously induced putatively homozygous (up to $21.1 \%$ in TME 204) and heterozygous (up to $89.5 \%$ in 60444 ) mutations in MePDS gene in these $\mathrm{T}_{0}$ events. All putatively homozygous, mono-allelic plants showed full albino phenotypes while putatively homozygous, bi-allelic plants were either full or partial albinos, with putatively heterozygous counterparts displaying partial albino phenotypes.

The very high efficiency of mono and bi-allelic gene editing achieved from two constructs across both cultivars studied equates with similar reports in potato (83\%) (Wang et al., 2015) and cotton (98-100\%) (Gao et al., 2017; Li et al., 2017). The surviving edited mutants showed persistence of altered phenotype across micropropagation cycles and under greenhouse conditions. These results indicate significant potential for successful application of gene-editing technologies for the development of cassava with improved farmer and consumer traits. There is need, however, to further develop CRISPR/Cas9 
gene-editing capacity for this crop. It is considered that the high efficiencies achieved here result, at least in part, from integration of the CRISPR/Cas9 and gRNA cassette. Continuous expression of the editing tools maximizes opportunity for production of mutations at the target site. Although a powerful tool for basic research, such an approach is not optimal for developing enhanced germplasm with value for cassava farmers. Segregating away the integrated T-DNA by sexual crossing in cassava is possible, but is not desirable due to the crop's heterozygous nature and associated in-breeding depression. It is important, therefore, to develop genome-editing capacity for cassava that does not require integration of the CRISPR tools. Such techniques include direct delivery into totipotent cells and use of ribonucleoprotein (RNP) (Woo et al., 2015; Zhang et al., 2017) as alternatives to Agrobacterium-mediated transgenesis. The expanding range of alternatives to Cas, such as Cpf1 (Zetsche et al., 2015), also brings increased flexibility for targeting genes, transcription factors, and their control elements. The MePDS knockout system described here generates easily detectable mutated events in a relatively short time frame of 6-8 weeks, and due to direct visualization of the phenotype, does not require sequencing to confirm editing at the target. It therefore provides a valuable method to facilitate rapid assessment and optimization of CRISPR/Cas9 and other genome-editing technologies in cassava.

\section{AUTHOR CONTRIBUTIONS}

JO designed the experiments, produced, and analyzed the gene edited plants and wrote the manuscript. TA and

\section{REFERENCES}

Bart, R. S., and Taylor, N. J. (2017). New opportunities and challenges to engineer disease resistance in cassava, a staple food of African small-holder farmers. PLOS Pathog. 13:e1006287. doi: 10.1371/journal.ppat.1006287

Brooks, C., Nekrasov, V., Lippman, Z. B., and Van Eck, J. (2014). Efficient gene editing in tomato in the first generation using the clustered regularly interspaced short palindromic repeats/CRISPR-associated9 system. Plant Physiol. 166, 1292-1297. doi: 10.1104/pp.114.247577

Ceballos, H., Iglesias, C. A., Perez, J. C., and Dixon, A. G. (2004). Cassava breeding: opportunities and challenges. Plant Mol. Biol. 56, 503-516.

Ceballos, H., Kawuki, R. S., Gracen, V. E., Yencho, G. C., and Hershey, C. H. (2015). Conventional breeding, marker-assisted selection, genomic selection and inbreeding in clonally propagated crops: a case study for cassava. Theor. Appl. Genet. 128, 1647-1667. doi: 10.1007/s00122-015-2555-4

Chandrasekaran, J., Brumin, M., Wolf, D., Leibman, D., Klap, C., Pearlsman, M., et al. (2016). Development of broad virus resistance in non-transgenic cucumber using CRISPR/Cas9 technology. Mol. Plant Pathol. 17, 1140-1153. doi: $10.1111 / \mathrm{mpp} .12375$

Chauhan, R. D., Beyene, G., Kalyaeva, M., Fauquet, C. M., and Taylor, N. (2015). Improvements in Agrobacterium-mediated transformation of cassava (Manihot esculenta Crantz) for large-scale production of transgenic plants. Plant Cell Tiss. Organ Cult. 121, 591-603. doi: 10.1007/s11240-015-0729-z

Curtin, S. J., Voytas, D. F., and Stupar, R. M. (2012). Genome engineering of crops with designer nucleases. Plant Genome 5, 42-50. doi: 10.3835/ plantgenome2012.06.0008

Ding, Y., Li, H., Chen, L. L., and Xie, K. (2016). Recent advances in genome editing using CRISPR/Cas9. Front. Plant Sci. 7:703. doi: 10.3389/fpls.2016.00703

Doudna, J. A., and Charpentier, E. (2014). Genome editing. The new frontier of genome engineering with CRISPR-Cas9. Science 346:1258096. doi: 10.1126/ science. 1258096
II interpreted the data and edited the manuscript. RB designed the analysis and interpreted sequencing data, wrote and edited the manuscript. DN designed the experiments and edited the manuscript. NT supervised the design of experiments and analysis of edited plants, wrote and edited the manuscript.

\section{FUNDING}

This work was supported by funds from the Bill and Melinda Gates Foundation.

\section{ACKNOWLEDGMENTS}

The authors thank B. Staskawicz and M. Gómez, UC Berkeley, CA, United States, for donating the plasmids and guidance on cloning, Getu Duguma for data interpretation support, Kira Veley for guidance with sequence data analysis, Deepika Chauhan for cassava transformation, and greenhouse staff at DDPSC for plant care.

\section{SUPPLEMENTARY MATERIAL}

The Supplementary Material for this article can be found online at: https://www.frontiersin.org/articles/10.3389/fpls.2017.01780/ full\#supplementary-material

Doyle, J. J., and Doyle, J. L. (1990). Isolation of plant DNA from fresh tissue. Focus 12, 13-15.

Fan, D., Liu, T., Li, C., Jiao, B., Li, S., Hou, Y., et al. (2015). Efficient CRISPR/Cas9mediated targeted mutagenesis in populus in the first generation. Sci. Rep. 5:12217. doi: $10.1038 /$ srep 12217

Gao, W., Long, L., Tian, X., Xu, F., Liu, J., Singh, P. K., et al. (2017). Genome editing in cotton with the CRISPR/Cas9 system. Front. Plant Sci. 8:1364. doi: 10.3389/fpls.2017.01364

Jia, H., and Wang, N. (2014). Targeted genome editing of sweet orange using Cas9/sgRNA. PLOS ONE 9:e93806. doi: 10.1371/journal.pone.0093806

Jiang, W., Zhou, H., Bi, H., Fromm, M., Yang, B., and Weeks, D. P. (2013). Demonstration of CRISPR/Cas9/sgRNA-mediated targeted gene modification in Arabidopsis, tobacco, sorghum and rice. Nucleic Acids Res. 41, e188. doi: $10.1093 /$ nar/gkt780

Johnson, R. D., and Jasin, M. (2001). Double-strand-break-induced homologous recombination in mammalian cells. Biochem. Soc. Trans. 29, 196-201.

Joung, J. K., and Sander, J. D. (2013). TALENs: a widely applicable technology for targeted genome editing. Nat. Rev. Mol. Cell Biol. 14, 49-55. doi: 10.1038/ nrm3486

Li, C., Unver, T., and Zhang, B. (2017). A high-efficiency CRISPR/Cas9 system for targeted mutagenesis in cotton (Gossypium hirsutum L.). Sci. Rep. 7:43902. doi: $10.1038 /$ srep43902

Li, J. F., Norville, J. E., Aach, J., Mccormack, M., Zhang, D., Bush, J., et al. (2013). Multiplex and homologous recombination-mediated genome editing in Arabidopsis and Nicotiana benthamiana using guide RNA and Cas9. Nat. Biotechnol. 31, 688-691. doi: 10.1038/nbt.2654

Nekrasov, V., Staskawicz, B., Weigel, D., Jones, J. D., and Kamoun, S. (2013). Targeted mutagenesis in the model plant Nicotiana benthamiana using Cas 9 RNA-guided endonuclease. Nat. Biotechnol. 31, 691-693. doi: 10.1038/nbt. 2655 
Nishitani, C., Hirai, N., Komori, S., Wada, M., Okada, K., Osakabe, K., et al. (2016). Efficient genome editing in apple using a CRISPR/Cas9 system. Sci. Rep. 6:31481. doi: 10.1038/srep31481

Pennisi, E. (2013). The CRISPR craze. Science 341, 833-836. doi: 10.1126/science. 341.6148 .833

Puchta, H., Dujon, B., and Hohn, B. (1996). Two different but related mechanisms are used in plants for the repair of genomic double-strand breaks by homologous recombination. Proc. Natl. Acad. Sci. U.S.A. 93, 5055-5060.

Qin, G., Gu, H., Ma, L., Peng, Y., Deng, X. W., Chen, Z., et al. (2007). Disruption of phytoene desaturase gene results in albino and dwarf phenotypes in Arabidopsis by impairing chlorophyll, carotenoid, and gibberellin biosynthesis. Cell Res. 17, 471-482. doi: 10.1038/cr.2007.40

Shan, Q., Wang, Y., Li, J., Zhang, Y., Chen, K., Liang, Z., et al. (2013). Targeted genome modification of crop plants using a CRISPR-Cas system. Nat. Biotechnol. 31, 686-688. doi: 10.1038/nbt.2650

Sonoda, E., Hochegger, H., Saberi, A., Taniguchi, Y., and Takeda, S. (2006). Differential usage of non-homologous end-joining and homologous recombination in double strand break repair. DNA Repair 5, 1021-1029. doi: 10.1016/j.dnarep.2006.05.022

Sun, X., Hu, Z., Chen, R., Jiang, Q., Song, G., Zhang, H., et al. (2015). Targeted mutagenesis in soybean using the CRISPR-Cas9 system. Sci. Rep. 5:10342. doi: $10.1038 /$ srep 10342

Svitashev, S., Schwartz, C., Lenderts, B., Young, J. K., and Mark Cigan, A. (2016). Genome editing in maize directed by CRISPR-Cas9 ribonucleoprotein complexes. Nat. Commun. 7:13274. doi: 10.1038/ncomms13274

Symington, L. S., and Gautier, J. (2011). Double-strand break end resection and repair pathway choice. Annu. Rev. Genet. 45, 247-271. doi: 10.1146/annurevgenet-110410-132435

Taylor, N., Gaitan-Solis, E., Moll, T., Trauterman, B., Jones, T., Pranjal, A., et al. (2012). A High-throughput platform for the production and analysis of transgenic cassava (Manihot esculenta) plants. Trop. Plant Biol. 5, 127-139. doi: 10.1007/s12042-012-9099-4

Wang, S., Zhang, S., Wang, W., Xiong, X., Meng, F., and Cui, X. (2015). Efficient targeted mutagenesis in potato by the CRISPR/Cas9 system. Plant Cell Rep. 34, 1473-1476. doi: 10.1007/s00299-015-1816-7

Woo, J. W., Kim, J., Kwon, S. I., Corvalan, C., Cho, S. W., Kim, H., et al. (2015). DNA-free genome editing in plants with preassembled CRISPR-Cas9 ribonucleoproteins. Nat. Biotechnol. 33, 1162-1164. doi: 10.1038/nbt.3389

Zetsche, B., Gootenberg, J. S., Abudayyeh, O. O., Slaymaker, I. M., Makarova, K. S., Essletzbichler, P., et al. (2015). Cpf1 is a single RNA-guided endonuclease of a class 2 CRISPR-Cas system. Cell 163, 759-771. doi: 10.1016/j.cell.2015.09.038

Zhang, H., Zhang, J., Wei, P., Zhang, B., Gou, F., Feng, Z., et al. (2014). The CRISPR/Cas9 system produces specific and homozygous targeted gene editing in rice in one generation. Plant Biotechnol. J. 12, 797-807. doi: 10.1111/pbi. 12200

Zhang, K., Raboanatahiry, N., Zhu, B., and Li, M. (2017). Progress in genome editing technology and its application in plants. Front. Plant Sci. 8:177. doi: $10.3389 /$ fpls.2017.00177

Conflict of Interest Statement: The authors declare that the research was conducted in the absence of any commercial or financial relationships that could be construed as a potential conflict of interest.

Copyright (C) 2017 Odipio, Alicai, Ingelbrecht, Nusinow, Bart and Taylor. This is an open-access article distributed under the terms of the Creative Commons Attribution License (CC BY). The use, distribution or reproduction in other forums is permitted, provided the original author(s) or licensor are credited and that the original publication in this journal is cited, in accordance with accepted academic practice. No use, distribution or reproduction is permitted which does not comply with these terms. 